Pre-print of full paper presented at CONVERSATIONS 2019 - an international workshop on chatbot research, November19-20, Amsterdam, the Netherlands. The final authenticated version of the paper is available online at https://doi.org/10.1007/978-3-030-39540-7_3

\title{
Privacy Concerns in Chatbot Interactions
}

\author{
Carolin Ischen $(-)$, Theo Araujo, Hilde Voorveld, Guda van Noort, and Edith Smit \\ ASCoR, University of Amsterdam, 1001 NG Amsterdam, Netherlands \\ \{C.Ischen, T.B.Araujo, H.A.M.Voorveld, G.vanNoort, E.G.Smit\}@uva.nl
}

\begin{abstract}
Chatbots are increasingly used in a commercial context to make product- or service-related recommendations. By doing so, they collect personal information of the user, similar to other online services. While privacy concerns in an online (website-) context are widely studied, research in the context of chatbot-interaction is lacking. This study investigates the extent to which chatbots with human-like cues influence perceptions of anthropomorphism (i.e., attribution of human-like characteristics), privacy concerns, and consequently, information disclosure, attitudes and recommendation adherence. Findings show that a human-like chatbot leads to more information disclosure, and recommendation adherence mediated by higher perceived anthropomorphism and subsequently, lower privacy concerns in comparison to a machine-like chatbot. This result does not hold in comparison to a website; human-like chatbot and website were perceived as equally high in anthropomorphism. The results show the importance of both mediating concepts in regards to attitudinal and behavioral outcomes when interacting with chatbots.
\end{abstract}

Keywords: Chatbots $\bullet$ Anthropomorphism $\bullet$ Privacy Concerns

\section{$1 \quad$ Introduction}

Through advances in artificial intelligence and machine learning, conversational agents in the form of text-based chatbots become more and more important for companies and brands to make product-, or service-related recommendations [18]. Chatbots interact with their users through natural language, and can provide guidance in a conversational manner [21, 42]. The conversational interaction in combination with human-like cues are crucial characteristics of such chatbots. Because of these characteristics, users might be more likely to attribute human-like characteristics to them (i.e., perceive them as anthropomorphic) $[17,27]$. While this might lead users to appreciate the dialog and enjoy the interaction $[10,26]$, they also need to share personal information with the chatbot to receive a valuable recommendation. By doing so, the company that hosts the chatbot collects data of their users, who are possibly not aware of this data collection. In this regard, chatbots might also enhance privacy concerns users might have when interacting with digital technologies [15]. While we know from previous research that users are concerned about their online privacy when using websites [e.g., 8], also 
having downstream effects on e.g. self-disclosure [9], users' privacy concerns might differ for chatbots, especially when conveying a human-like appeal.

Therefore, this study investigates to what extent privacy concerns in chatbot interactions are related to users' attitudes and recommendation adherence, and furthermore, to what extent users feel comfortable sharing personal information with a human-like chatbot in comparison with a machine-like chatbot, or a website. The research question guiding this research is: To what extent do human-like characteristics of a chatbot influence perceived anthropomorphism, privacy concerns, and consequently, information disclosure, attitudes, and recommendation adherence?

Hereby, building on social response theory and previous research on informational privacy, this study aims to examine anthropomorphism and privacy concerns as sequential underlying mechanisms possibly explaining these outcomes. This study contributes to our understanding of chatbots in a digital communication environment in exploring how human-like attributes influence users' perceptions of the communication entity (chatbot and website) and their behavior when interacting with them. In examining the concept of privacy concerns, this study not only extends research in the field of humanmachine-communication, but has societal implications. While the protection of online privacy is widely discussed, the acceptance of chatbots and related implications for privacy still need to be studied. It plays an increasingly important role to uncover whether and, if so, how human-like cues influence privacy concerns, and how aware users are that their data is being used. This specific context has to be addressed by companies using this technology as well as by policy makers to protect users' privacy.

\section{Theoretical Background}

\subsection{Perceived Anthropomorphism}

Anthropomorphism is the attribution of human-like characteristics to non-human entities [17]. This can be mindful, i.e. the conscious evaluation of humanness, as well as mindless, i.e. attributing human-like characteristics without realizing, such as being friendly or sociable [27]. Go and Sundar [20] differentiate three types of cues that can suggest humanness among chatbots (mindful as well as mindless). These are visual cues, such as the use of human-like figures; identity cues, such as human-associated names; and conversational cues, such as the mimicking of human language, i.e. acknowledging responses. The authors find effects of conversational cues on attitudes and behavioral intentions, and further, interaction effects for the three types of cues. A combination of the anthropomorphic cues as used in a human-like chatbot is expected to have the ability to influence mindless evaluations of humanness in particular, both in comparison to a machine-like chatbot without these anthropomorphic cues, and in comparison to a more traditional form of digital medium like an interactive website [2]. We do not expect differences between a machine-like chatbot and an interactive website. This is because both, website and machine-like chatbot include interactive elements (e.g., people have to disclose some personal information by answering questions) that might create a similar perception of anthropomorphism [26]. 
Subsequently, we examine three different outcomes. Firstly, level of comfort with information disclosure will be studied. Information disclosure in this context is the amount of true information customers reveal about themselves for a purchase decision online, e.g. providing personal details [9]. Level of comfort with information disclosure refers to user perceptions, i.e., to what extent they feel like having disclosed private or intimate information about themselves. Secondly, we will study attitudes towards the entity giving the recommendation, in this case the chatbot/website. The third outcome variable is recommendation adherence, i.e., the willingness to purchase the recommended product. We expect perceived anthropomorphism to positively influence all three outcome variables. In accordance with social response theory, stating that humans respond similarly to technology imbued with human-like characteristics as they respond to humans [30,33], Go and Sundar [20] showed that attitudes and behavioral intentions can be influenced by the social connectedness induced by anthropomorphism. Secondly, based on brand relationship theory, users and brands can engage in relationships similar to interpersonal relationships, leading to positive brand responses [19, 31]. Similarly, interacting with a human-like chatbot created by a company can mimic interpersonal communication, positively influencing information disclosure, attitudes and recommendation adherence. Therefore, we propose the following hypothesis (H1):

Receiving a product recommendation from a human-like chatbot leads to more (a) information disclosure, (b) positive attitudes towards the medium, and (c) recommendation adherence mediated by higher perceived anthropomorphism than receiving a product recommendation from a machine-like chatbot or an interactive website.

\subsection{Privacy Concerns}

Privacy concerns in a marketing context can be defined as "the degree to which a consumer is worried about the potential invasion of the right to prevent the disclosure of personal information to others" $[3,37]$. To make recommendations, online services collect personal information. Personal information can amongst others be collected by direct requests to disclose this information. These direct requests might induce privacy concerns [39]. Previous research by Følstad et al. [18] showed that customers have a concern for privacy and security when it comes to interactions with chatbots, i.e. they have a need to be provided with a secure online service. As the conversation with a chatbot is a rather new phenomenon, users might be more aware of these direct requests, inducing more privacy concerns.

We expect privacy concerns to negatively influence information disclosure. While, as stated in the "privacy paradox", perceived privacy risks do not automatically translate into not disclosing information online $[4,14]$, several scholars found privacy concerns and information disclosure to be related [5]. For example, Dinev and Hart [15] found a negative relationship between privacy concerns and the willingness to provide personal information for internet-based transactions. Furthermore, privacy concerns are an important antecedent for the acceptance of mobile chatbots [16], and might thus be related to users' attitudes. Thirdly, users see privacy concerns as a reason to not shop online [23]. Hence, we expect privacy concerns to also negatively influence 
recommendation adherence. However, since we cannot formulate a direction of how human-like cues influence privacy concerns, we propose the following research question (RQ1):

Does receiving a product recommendation from a human-like chatbot lead to more, or less (a) information disclosure, (b) positive attitudes towards the medium, and (c) recommendation adherence mediated by higher privacy concerns than receiving a product recommendation from a machine-like chatbot or an interactive website?

\subsection{Sequential Mediation of Perceived Anthropomorphism and Privacy Concerns}

Lastly, we are interested in the relationship between perceived anthropomorphism and privacy concerns in a chatbot context. On the one hand, we argue that a chatbot that is perceived as highly anthropomorphic can enhance privacy concerns, when asking to disclose personal information. A human-like chatbot as a communication entity might be perceived as more personal and less anonymous, inducing more privacy concerns than a machine-like chatbot or a website $[22,35]$. On the other hand, this feeling of communicating with an actual communication partner might also lead to less privacy concerns, because users might experience a closer connection to the human-like chatbot, increasing the willingness to use it as a companion [7]. Research in the health context showed that a chatbot was evaluated positively in comparison to e.g. search engines by adolescents, especially in regards to more sensitive questions [12]. Due to these contradictory findings, we propose a research question about the sequential mediating effects of perceived anthropomorphism and privacy concerns (RQ2):

Does receiving a product recommendation from a human-like chatbot lead to more, or less (a) information disclosure, (b) positive attitudes towards the medium, and (c) recommendation adherence sequentially mediated by perceived anthropomorphism and privacy concerns than receiving a product recommendation from a machine-like chatbot or an interactive website?

\section{$3 \quad$ Method}

\subsection{Design and Sample}

An experimental between-subjects design with three conditions (type of entity: humanlike chatbot vs. machine-like chatbot vs. website) was implemented. Recruited through the Dutch online panel PanelClix, 231 participants took part in the study. Participants' age ranged from 18 to $73(M=41.83, S D=14.01), 48.5 \%$ were female ( $51.5 \%$ male); $51.6 \%$ indicated to have a high educational level (38.9\% middle, $9.5 \%$ low).

\subsection{Procedure}

Randomly assigned to the groups, participants interacted with either the website $\left(n_{\text {website }}\right.$ $=73)$, the machine-like $\left(n_{\text {machine-like }}=85\right)$, or the human-like $\left(n_{\text {human-like }}=73\right)$ chatbot to 
obtain a recommendation for the (fictitious) health insurance company "ZorgPlus" (engl. "CarePlus"). Several questions about demographics (i.e., age, gender, place of residence), preferences (i.e., current health insurance company, importance of customer service and travel behavior, budget), and two intrusive questions (i.e., legal residence in the Netherlands and number of sexual partners in the previous six-month) were asked, that participants could answer as they wish. Afterwards, participants filled in a questionnaire measuring the dependent variables, mediators and control variables.

\subsection{Stimuli}

An interactive website was developed that gave a recommendation for a health insurance after participants filled in personal information. ${ }^{1}$ Furthermore, two chatbots were created for this study using a conversational agent research toolkit for experimental research developed by Araujo [1]. The human-like version of the chatbot introduced itself with a name ("Sam"); displayed a visual of a cartoon-like customer service agent, similar to Verhagen et al. [36]; and used human conversational cues, i.e. acknowledged the responses of the participants (e.g. "gotcha", "I noted down your gender"). In the machine-like version, the chatbot did not carry a human-like name (it was called ChatbotX), similar to Araujo [2]; displayed a neutral visual of a dialog bubble, similar to Go and Sundar [20]; and only asked questions without acknowledging previous answers. An example of the human-like chatbot is given in Figure 1.

Figure 1. Stimulus Material Human-like Chatbot

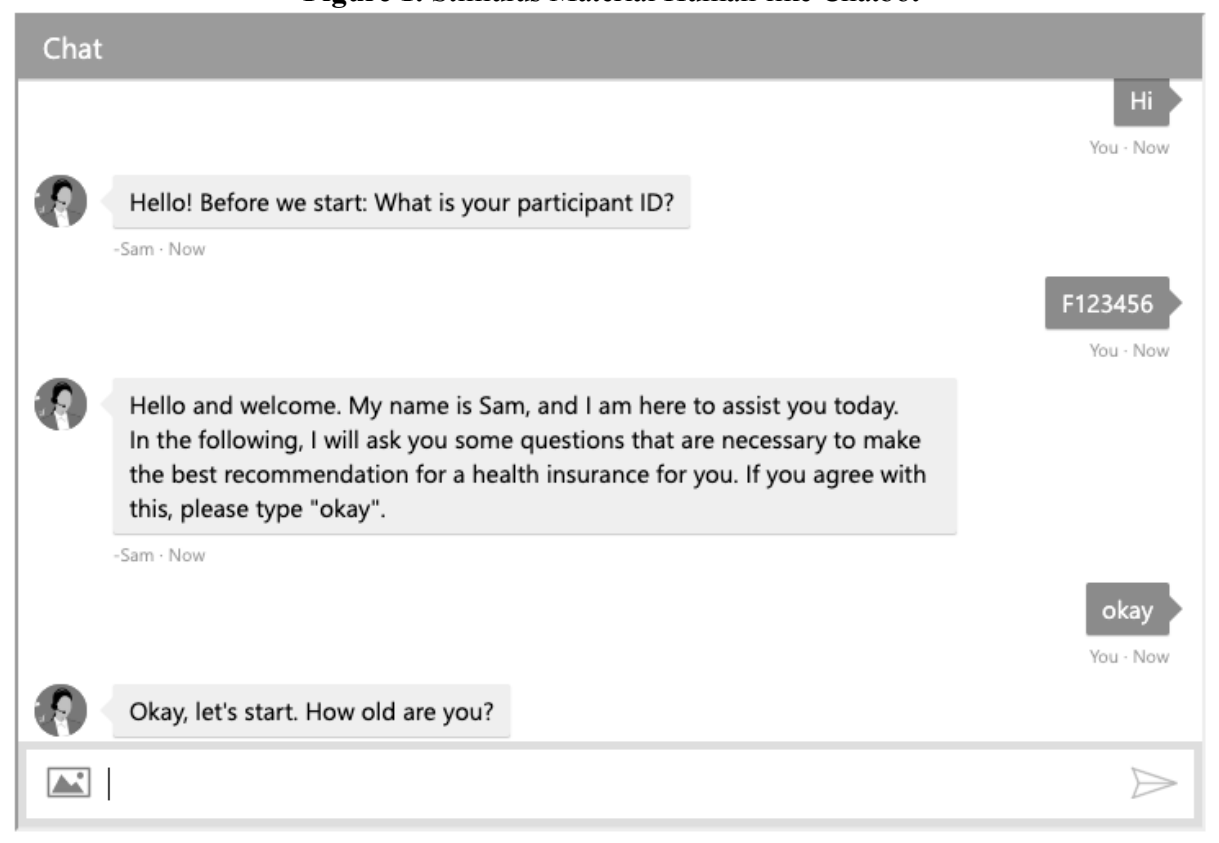

${ }^{1}$ Similar to [26], conference presentation is available upon request to the first author. 


\subsection{Measurements}

Mediators. We measured mindless anthropomorphism with four items on a 7-pointLikert-scale adapted from Kim and Sundar [27], e.g., "I perceived the chatbot/website as sociable" $(1=$ strongly disagree, $7=$ strongly agree $){ }^{2}$ Privacy concerns were measured with four items on a 7-point-Likert-scale including "It bothers me that this chatbot asks me for this much personal information" ( $1=$ strongly disagree, $7=$ strongly agree $)$ adapted from Xu et al. [40]. While the original scale was developed to measure privacy concerns as a trait, we adapted the measurement to assess privacy concerns in regards to the specific interaction participants engaged in.

Outcome Variables. Level of comfort with information disclosure was measured with four items adapted from Croes and Antheunis [11,28], e.g. "I felt comfortable disclosing personal information during the interaction" $(1=$ strongly disagree, 7 = strongly agree). Attitude towards the chatbot/website was measured with items adapted from Becker-Olsen [6]. Five semantic differential scale items were used e.g "I think the chatbot/website is good/bad". To measure recommendation adherence, we used four items on a 7-point-Likert-scale adapted from Dabholkar and Sheng [13] including "Imagine you are considering a new health insurance: It is very likely that I would buy the recommended insurance" ( $1=$ strongly disagree, $7=$ strongly agree $)$.

Control Variables. Besides age, gender, and education, we measured familiarity (with the chatbot/website, and with health insurances) with two items on a 7-point-Likertscale adapted from Zhou, Yang \& Hui [43]. Furthermore, we measured power usage [29], belief in machine heuristic [34], and enjoyment [23] as control variables. Scale reliabilities and mean values of the relevant scales are displayed in Table 1.

Table 1. Scale Reliability

\begin{tabular}{llll}
\hline Scale & Cr. Alpha & M & SD \\
\hline Mindless anthropomorphism & .91 & 4.55 & 1.21 \\
Privacy Concerns & .91 & 4.20 & 1.28 \\
Information Disclosure & .89 & 4.24 & 1.15 \\
Attitudes & .92 & 4.45 & 1.20 \\
Recommendation Adherence & .83 & 3.56 & 1.01 \\
Familiarity Medium & .83 & 4.76 & 1.39 \\
\hline
\end{tabular}

\subsection{Randomization Check}

A randomization check showed that participants did not differ across groups in terms of age, gender, education, power usage, enjoyment, belief in machine heuristic, and familiarity with health insurances. Significant differences were found for familiarity

2 Furthermore, we measured mindful anthropomorphism with three items on 7-point semantic differential scales [32]. A univariate analysis of variance showed no significant main effect of type of entity on mindful anthropomorphism $(F(2,227)=1.16, \mathrm{p}=.314)$. 
with the medium $(F(2,228)=15.79, p<.001)$. Participants were significantly less familiar with chatbots $\left(M_{\text {machine-like }}=4.49, S D=1.37, M_{\text {human-like }}=4.36, S D=1.40\right)$ than with $\left(M_{\text {websites }}=5.47, S D=1.12\right)$. Familiarity with the medium was included as a covariate in the subsequent analyses.

\section{$4 \quad$ Results}

We performed serial multiple mediation analyses (model 6), using the PROCESS macro for IBM SPSS version 25 [24]. We used bootstrapping (5,000 bootstrap samples) to obtain bias-corrected $95 \%$ confidence intervals for the indirect effects of the independent variable type of entity on information disclosure, attitudes, and recommendation adherence through the mediators mindless anthropomorphism and privacy concerns. All paths for the full model are shown in Figure 1 and the corresponding coefficients are displayed in Tables 2-4. Separate path analyses were performed for the three dependent variables. The independent variable type of entity is multicategorical. We use the category human-like chatbot as the reference category, since we are primarily interested in the comparison human-like chatbot vs. machine-like chatbot, and humanlike chatbot vs. website [25]. Additionally, we also compared machine-like chatbot and website using the category machine-like chatbot as the reference category.

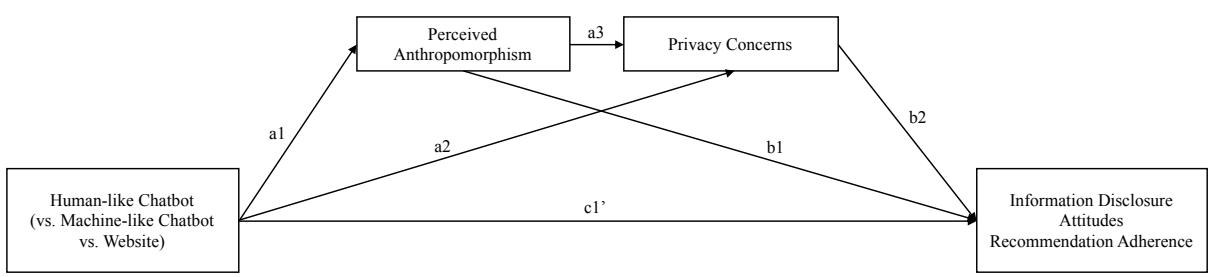

Fig. 1. Serial mediation model

\subsection{Information Disclosure}

Perceived Anthropomorphism and Information Disclosure. Firstly, we compared human-like chatbot to machine-like chatbot. As shown in Table 2, there is a significant direct effect of type of entity on mindless anthropomorphism (path a1). The human-like chatbot is perceived as higher in anthropomorphism than the machine-like chatbot. The specific indirect effect of type of entity on information disclosure through mindless anthropomorphism is significant (effect $=-.20, S E=.08, \mathrm{CI}=-.37,-.06$ ), indicating that the human-like chatbot is perceived as higher in anthropomorphism than the machine-like chatbot, leading to more information disclosure. Secondly, comparing the human-like chatbot and the website, no significant effect on perceived anthropomorphism (path a1) is found, both are equally high in anthropomorphism. The specific indirect effect of type of entity on information disclosure through mindless anthropomorphism is not significant (effect $=-.12, S E=.07, \mathrm{CI}=-.28, .01$ ), indicating no mediating effect of anthropomorphism. Additionally, we also compared machine-like chatbot and website, showing no significant effect on perceived anthropomorphism. The specific 
indirect effect of type of entity on information disclosure through mindless anthropomorphism is not significant (effect $=.08, S E=.07, \mathrm{CI}=-.06, .22$ ).

Privacy Concerns and Information Disclosure. Firstly, we compared human-like chatbot to machine-like chatbot. There is no significant direct effect of type of entity on privacy concerns (path a2). The specific indirect effect on information disclosure through privacy concerns is not significant (effect $=.07, S E=.07, \mathrm{CI}=-.06, .21$ ). Secondly, comparing the human-like chatbot and the website, no significant effect on privacy concerns (path a2) is found. The specific indirect effect on information disclosure through privacy concerns is also not significant (effect $=.08, S E=.29, \mathrm{CI}=-.29$, .02). Thirdly, comparing machine-like chatbot and website, there is a significant direct effect on privacy concerns. Furthermore, the specific indirect effect of type of entity through privacy concerns is significant (effect $=-.20, S E=.08, \mathrm{CI}=-.36,-.06$ ), indicating a mediating effect. The website induced higher privacy concerns than the machine-like chatbot, leading to less information disclosure.

Perceived Anthropomorphism, Privacy Concerns and Information Disclosure. Firstly, we tested a serial multiple mediation (including perceived anthropomorphism, and privacy concerns) comparing human-like chatbot and machine-like chatbot. The specific indirect effect of type of entity on information disclosure through both, mindless anthropomorphism and privacy concerns is significant (effect $=-.04, S E=.02, \mathrm{CI}$ $=-.09,-.01)$. The human-like chatbot is perceived as higher in mindless anthropomorphism than the machine-like chatbot, leading to less privacy concerns, and consequently more information disclosure. Secondly, testing a serial multiple mediation comparing human-like chatbot and website, the specific indirect effect of type of entity on information disclosure through both, mindless anthropomorphism and privacy concerns is not significant (effect $=-.03, S E=.02, \mathrm{CI}=-.07, .001$ ). Thirdly, testing a serial multiple mediation comparing machine-like chatbot and website, the specific indirect effect of type of entity on information disclosure through both, mindless anthropomorphism and privacy concerns is not significant (effect $=.02, S E=.02, \mathrm{CI}=-.01, .05$ ).

Table 2. Path coefficients sequential mediation explaining information disclosure

\begin{tabular}{rrrr}
\hline & $\begin{array}{c}\text { Human-like chatbot vs. } \\
\text { machine-like chatbot }\end{array}$ & $\begin{array}{r}\text { Human-like chatbot vs. } \\
\text { website }\end{array}$ & $\begin{array}{r}\text { Machine-like chatbot } \\
\text { vs. website }\end{array}$ \\
\hline a1 & $-.51^{* *}(.18)$ & $-.32(.19)$ & $.20(.18)$ \\
a2 & $-.20(.20)$ & $.37(.21)$ & $.56^{*}(.20)$ \\
a3 & $-p e r .24^{* *}(.07)$ & $-.24^{* *}(.07)$ & $-.24^{* *}(.07)$ \\
b1 & $.39^{* * *}(.05)$ & $.39^{* * *}(.05)$ & $.39^{* * *}(.05)$ \\
b2 & $-.35^{* * *(.05)}$ & $-.35^{* * *(.05)}$ & $-.35^{* * *(.05)}$ \\
c1 & $-.10(.13)$ & $-.02(.14)$ & $.08(.14)$ \\
\hline$* \mathrm{p}=.05 . * * \mathrm{p}<.005 . * * * \mathrm{p}<.001 ;$ controlled for familiarity with medium
\end{tabular}




\subsection{Attitudes}

Perceived Anthropomorphism and Attitudes. The same type of analysis was conducted for attitudes as the outcome variable, as shown in Table 3. Firstly, we compared human-like chatbot and machine-like chatbot. The specific indirect effect through mindless anthropomorphism is significant (effect $=-.33, S E=.11, \mathrm{CI}=-.56,-.11$ ), indicating that the human-like chatbot is perceived as higher in anthropomorphism than the machine-like chatbot, leading to more positive attitudes. When comparing the human-like chatbot and the website, the specific indirect effect through mindless anthropomorphism is not significant (effect $=-.20, S E=.11, \mathrm{CI}=-.43, .01$ ). Lastly, comparing machine-like chatbot and website, the specific indirect effect through mindless anthropomorphism is also not significant (effect $=.13, S E=.12, \mathrm{CI}=-.10, .36$ ).

Privacy Concerns and Attitudes. When comparing human-like chatbot and machinelike chatbot, the specific indirect effect through privacy concerns is not significant (effect $=.01, S E=.02, \mathrm{CI}=-.01, .06$ ). The same holds for the comparison human-like chatbot and website (effect $=-.02, S E=.03, \mathrm{CI}=-.09, .02$ ); and machine-like chatbot and website (effect $=-.04, S E=.03, \mathrm{CI}=-.11, .02)$.

Perceived Anthropomorphism, Privacy Concerns, and Attitudes. When testing a serial multiple mediation comparing human-like chatbot and machine-like chatbot, the specific indirect effect of type of entity on attitudes through both, mindless anthropomorphism and privacy concerns is not significant (effect $=-.01, S E=.01, \mathrm{CI}=-.03$, $.004)$. The same holds for the serial multiple mediation models comparing human-like chatbot and website (effect $=-.01, S E=.01, \mathrm{CI}=-.02, .003$ ); and machine-like chatbot and website (effect $=.003, S E=.004, \mathrm{CI}=-.004, .03)$.

Table 3. Path coefficients sequential mediation explaining attitudes

\begin{tabular}{crrr}
\hline & $\begin{array}{c}\text { Human-like vs. ma- } \\
\text { chine-like }\end{array}$ & Human-like vs. website & $\begin{array}{c}\text { Machine-like vs. web- } \\
\text { site }\end{array}$ \\
\hline a1 & $-.51^{* *}(.18)$ & $-.32(.19)$ & $.20(.18)$ \\
a2 & $-.20(.20)$ & $.37(.21)$ & $.56 *(.20)$ \\
a3 & $-.24^{* *}(.07)$ & $-.24^{* *}(.07)$ & $-.24^{* *}(.07)$ \\
b1 & $.64^{* * *(.05)}$ & $.64 * * *(.05)$ & $.64 * * *(.05)$ \\
b2 & $-.07(.05)$ & $-.07(.05)$ & $-.07(.05)$ \\
c1 & $.15(.14)$ & $.10(.15)$ & $-.05(.15)$ \\
\hline$* \mathrm{p}=.05 . * * \mathrm{p}<.005 . * * * \mathrm{p}<.001 ;$ controlled for familiarity with medium
\end{tabular}

\subsection{Recommendation adherence}

Perceived Anthropomorphism and Recommendation Adherence. We tested the models for recommendation adherence as the outcome variable, as shown in Table 4. Firstly, we compared human-like chatbot to machine-like chatbot. The specific indirect effect through mindless anthropomorphism is significant (effect $=-.16, S E=.06, \mathrm{CI}=$ $-.29,-.05)$, indicating that the human-like chatbot is perceived as higher in 
anthropomorphism than the machine-like chatbot, leading to more recommendation adherence. When comparing the human-like chatbot and the website, the specific indirect effect through mindless anthropomorphism is not significant (effect $=-.10, S E=.06$, $\mathrm{CI}=-.22, .01)$. Lastly, comparing machine-like chatbot and website, the specific indirect effect through mindless anthropomorphism is not significant (effect $=.06, S E=$ $.06, \mathrm{CI}=-.05, .19)$.

Privacy Concerns and Recommendation Adherence. When comparing human-like chatbot and machine-like chatbot, the specific indirect effect through privacy concerns is not significant (effect $=.03, S E=.03, \mathrm{CI}=-.02, .09$ ). When comparing the humanlike chatbot and the website, the specific indirect effect through privacy concerns is not significant (effect $=-.05, S E=.04, \mathrm{CI}=-.13, .01$ ). Comparing the machine-like chatbot and the website, the specific indirect effect through privacy concerns is significant (effect $=-.07, S E=.04, \mathrm{CI}=-.17,-.004)$. The website induces higher privacy concerns than the machine-like chatbot, leading to less recommendation adherence.

Perceived Anthropomorphism, Privacy Concerns, and Recommendation Adherence. When testing a serial multiple mediation comparing human-like chatbot and machine-like chatbot, the specific indirect effect of type of entity on information disclosure through both, mindless anthropomorphism and privacy concerns is significant (effect $=-.02, \mathrm{SE}=.01, \mathrm{CI}=-.04,-.002)$. The human-like chatbot is perceived as higher in anthropomorphism than the machine-like chatbot, leading to less privacy concerns, and consequently more recommendation adherence. When testing the serial multiple mediation comparing the human-like chatbot and the website, the specific indirect effect of type of entity on information disclosure through both, mindless anthropomorphism and privacy concerns is not significant (effect $=-.01, S E=.01, \mathrm{CI}=-.03, .0008$ ). The same holds for the comparison machine-like chatbot and website (effect $=.01, S E=.01$, CI $=-.01, .02)$. A summary of the results is given in Table 5 .

Table 4. Path coefficients sequential mediation explaining recommendation adherence

\begin{tabular}{lrrr}
\hline & $\begin{array}{c}\text { Human-like vs. ma- } \\
\text { chine-like }\end{array}$ & Human-like vs. website & $\begin{array}{c}\text { Machine-like vs. web- } \\
\text { site }\end{array}$ \\
\hline a1 & $-.51^{* *}(.18)$ & $-.32(.19)$ & $.20(.18)$ \\
a2 & $-.20(.20)$ & $.37(.21)$ & $.56^{*}(.20)$ \\
a3 & $-.24^{* *}(.07)$ & $-.24^{* *}(.07)$ & $-.24 * *(.07)$ \\
b1 & $.32^{* * *}(.06)$ & $.32^{* * *}(.06)$ & $.32^{* * *}(.06)$ \\
b2 & $-.13^{* *}(.05)$ & $-.13^{* *}(.05)$ & $-.13^{* *}(.05)$ \\
c1 & $.25(.14)$ & $.30(.16)$ & $.05(.15)$ \\
\hline$* \mathrm{p}=.05 . * * \mathrm{p}<.005 . * * * \mathrm{p}<.001 ;$ controlled for familiarity with medium
\end{tabular}

Table 5. Summary of results

\begin{tabular}{llll}
\hline Hypothesis/RQ & $\begin{array}{c}\text { Human-like vs. machine } \\
\text { like chatbot }\end{array}$ & $\begin{array}{c}\text { Human-like chatbot } \\
\text { vs. website }\end{array}$ & $\begin{array}{c}\text { Machine-like chat- } \\
\text { bot vs. website }\end{array}$ \\
\hline $\begin{array}{l}\text { H1: Human-like } \\
\text { chatbot } \rightarrow\end{array}$ & $\begin{array}{l}\text { Supported. Human-like } \\
\text { chatbot leads to more }\end{array}$ & $\begin{array}{l}\text { Not supported. Dif- } \\
\text { ferences in }\end{array}$ & $\begin{array}{l}\text { Differences in an- } \\
\text { thropomorphism }\end{array}$
\end{tabular}




\begin{tabular}{|c|c|c|c|}
\hline $\begin{array}{l}\text { perceived anthro- } \\
\text { pomorphism } \rightarrow \\
\text { information dis- } \\
\text { closure/atti- } \\
\text { tudes/recommen- } \\
\text { dation adherence }\end{array}$ & $\begin{array}{l}\text { information disclosure, } \\
\text { positive attitudes and } \\
\text { recommendation adher- } \\
\text { ence mediated by higher } \\
\text { perceived anthropomor- } \\
\text { phism in comparison to } \\
\text { machine-like chatbot. }\end{array}$ & $\begin{array}{l}\text { anthropomorphism } \\
\text { between human-like } \\
\text { chatbot and website } \\
\text { not significant. }\end{array}$ & $\begin{array}{l}\text { between machine- } \\
\text { like chatbot and } \\
\text { website not signifi- } \\
\text { cant. }\end{array}$ \\
\hline $\begin{array}{l}\text { RQ1: Human-like } \\
\text { chatbot } \rightarrow \text { privacy } \\
\text { concerns } \rightarrow \text { infor- } \\
\text { mation disclo- } \\
\text { sure/attitudes/rec- } \\
\text { ommendation ad- } \\
\text { herence }\end{array}$ & $\begin{array}{l}\text { Not supported. Differ- } \\
\text { ences in privacy con- } \\
\text { cerns between human- } \\
\text { like chatbot and ma- } \\
\text { chine-like chatbot not } \\
\text { significant. }\end{array}$ & $\begin{array}{l}\text { Not supported. Dif- } \\
\text { ferences in privacy } \\
\text { concerns between } \\
\text { human-like chatbot } \\
\text { and website not sig- } \\
\text { nificant. }\end{array}$ & $\begin{array}{l}\text { Website induced } \\
\text { higher privacy con- } \\
\text { cerns than machine- } \\
\text { like chatbot, leading } \\
\text { to less information } \\
\text { disclosure, and less } \\
\text { recommendation } \\
\text { adherence. No me- } \\
\text { diating effect on at- } \\
\text { titudes. }\end{array}$ \\
\hline $\begin{array}{l}\text { RQ2: Human-like } \\
\text { chatbot } \rightarrow \text { per- } \\
\text { ceived anthropo- } \\
\text { morphism } \rightarrow \text { pri- } \\
\text { vacy concern } \rightarrow \\
\text { information dis- } \\
\text { closure/atti- } \\
\text { tudes/recommen- } \\
\text { dation adherence }\end{array}$ & $\begin{array}{l}\text { Partially supported. Hu- } \\
\text { man-like chatbot leads } \\
\text { to higher information } \\
\text { disclosure, and recom- } \\
\text { mendation adherence } \\
\text { mediated by higher an- } \\
\text { thropomorphism and } \\
\text { lower privacy concerns } \\
\text { than machine-like chat- } \\
\text { bot. No mediating effect } \\
\text { on attitudes. }\end{array}$ & $\begin{array}{l}\text { Not supported, no } \\
\text { sequential media- } \\
\text { tion. }\end{array}$ & $\begin{array}{l}\text { No sequential medi- } \\
\text { ation. }\end{array}$ \\
\hline
\end{tabular}

\section{$5 \quad$ Discussion and Conclusion}

This study investigated the extent to which chatbots' human-like characteristics influence perceived anthropomorphism, users' privacy concerns, and consequently, information disclosure, attitudes, and recommendation adherence. Firstly, we showed that a chatbot using human-like cues leads to higher mindless anthropomorphism than a chatbot not using these cues. Thus, people indeed attribute human-like characteristics such as friendliness or socialness to a chatbot.

Interestingly, mindless anthropomorphism was at the same level for the human-like chatbot and for the website. This result is puzzling, because it shows that interactive websites are (equally) able to convey a human-like appeal. This is in line with previous research showing no differences in, or even higher perceptions of anthropomorphism for websites than for chatbots [26, 38]. One possible explanation could be the source orientation. In the website condition, participants might have responded towards a source behind the website (company, programmer etc.), thus did not see the chatbot as an entity, but saw the "human behind", leading to higher perceived anthropomorphism. Another possible explanation is that users might change their "reference category" when interacting with a chatbot. While evaluating technology when comparing websites to one another, users might compare a chatbot with a human communication 
partner (including how a human would act), thus changing their expectations towards the chatbot. One important aspect might also be the familiarity with the medium. Users are more familiar with websites than with chatbots, which might have given them a sense of comfort already [41]. In this study, we found a significant difference in mindless anthropomorphism between the machine-like chatbot and the website when not controlling for familiarity. Participants might have given lower scores for friendliness or socialness for the machine-like chatbot because they were less familiar with the medium.

Furthermore, this research shows that the outcomes information disclosure, and recommendation adherence are indeed influenced by privacy concerns, supporting previous findings $[16,23]$. The sequential mediation including perceived anthropomorphism and privacy concerns shows that a human-like chatbot in this study is higher in perceived anthropomorphism, leading to less privacy concerns and subsequently, more comfort with disclosure, and more recommendation adherence. Users might experience a closer bond with a human-like agent than with a machine-like agent [7]. This might be because a human-like chatbot acknowledges users' answers, e.g. the chatbot in this study indicated that it "noted the answer down". This might have been perceived as less invading then just submitting it "somewhere" without knowing where the information ends up. No mediation was found for the comparison of human-like chatbot and website. These findings complement and extend a recent study [34] showing that users were more likely to reveal information to a machine-like interface than to a sales associate. Based on a machine-heuristic, users perceive a machine-like source as less biased. While these findings are based on source characteristics, our study focuses on message characteristics. Future research should look into the interplay of these two elements.

Additional analyses with a comparison of machine-like chatbot and website showed no influence of perceived anthropomorphism, but a direct effect on privacy concerns (while this direct effect is not significant for the other comparisons). This shows that a website is significantly higher in privacy concerns than a machine-like chatbot and that privacy concerns directly mediate information disclosure, attitudes and recommendation adherence. Further research should thus look into different underlying mechanisms apart from anthropomorphism.

Concluding, this study enriches our understanding of privacy concerns in a chatbot context in showing the sequential influence of perceived anthropomorphism and privacy concerns on users' behavioral intentions.

\section{References}

1. Araujo, T.: Conversational Agent Research Toolkit An alternative for creating and managing chatbots for experimental research. (2019). https://doi.org/https://doi.org/10.31235/osf.io/9ukyf.

2. Araujo, T.: Living up to the chatbot hype: The influence of anthropomorphic design cues and communicative agency framing on conversational agent and company perceptions. Comput. Human Behav. 85, 183-189 (2018). https://doi.org/10.1016/j.chb.2018.03.051.

3. Baek, T.H., Morimoto, M.: Stay Away From Me. J. Advert. 41, 1, 59-76 (2012). 
https://doi.org/10.2753/JOA0091-3367410105.

4. Barnes, S.B.: A privacy paradox: Social networking in the United States. First Monday. 11, 9, (2006).

5. Baruh, L. et al.: Online Privacy Concerns and Privacy Management: A Meta-Analytical Review. J. Commun. 67, 1, 26-53 (2017). https://doi.org/10.1111/jcom.12276.

6. Becker-Olsen, K.L.: And Now, A Word from our Sponsor: A Look at the Effects of Sponsored Content and Banner Advertising. J. Advert. 32, 2, 17-32 (2003).

7. Birnbaum, G.E. et al.: What robots can teach us about intimacy: The reassuring effects of robot responsiveness to human disclosure. Comput. Human Behav. 63, 416-423 (2016). https://doi.org/10.1016/j.chb.2016.05.064.

8. Boerman, S.C. et al.: Exploring Motivations for Online Privacy Protection Behavior: Insights From Panel Data. Communic. Res. (2018). https://doi.org/10.1177/0093650218800915.

9. Bol, N. et al.: Understanding the Effects of Personalization as a Privacy Calculus: Analyzing Self-Disclosure Across Health, News, and Commerce Contexts. J. Comput. Commun. 23, 370-388 (2018). https://doi.org/10.1093/jcmc/zmy020.

10. Chung, M. et al.: Chatbot e-service and customer satisfaction regarding luxury brands. J. Bus. Res. November 2017, 1-9 (2018). https://doi.org/10.1016/j.jbusres.2018.10.004.

11. Croes, E., Antheunis, M.L.: Can We Be Friends with a Chatbot? A Longitudinal Study on the Process of Friendship Formation Between Humans and a Social Chatbot. In: 69th Annual International Communication Association (ICA) Conference. (2019).

12. Crutzen, R. et al.: An artificially intelligent chat agent that answers adolescents' questions related to sex, drugs, and alcohol: An exploratory study. J. Adolesc. Heal. 48, 5, 514-519 (2011). https://doi.org/10.1016/j.jadohealth.2010.09.002.

13. Dabholkar, P.A., Sheng, X.: Consumer participation in using online recommendation agents: Effects on satisfaction, trust, and purchase intentions. Serv. Ind. J. 32, 9, 14331449 (2012). https://doi.org/10.1080/02642069.2011.624596.

14. Dienlin, T., Trepte, S.: Is the privacy paradox a relict of the past? An in-depth analysis of privacy attitudes and privacy behaviors. Eur. J. Soc. Psychol. 45, 285-297 (2015). https://doi.org/10.1002/ejsp.2038.

15. Dinev, T., Hart, P.: An extended privacy calculus model for e-commerce transactions. Inf. Syst. Res. 17, 1, 61-80 (2006). https://doi.org/10.1287/isre.1060.0080.

16. Van Eeuwen, M.: Mobile conversational commerce: messenger chatbots as the next interface between businesses and consumers. Univ. Twente. (2017).

17. Epley, N. et al.: On Seeing Human: A Three-Factor Theory of Anthropomorphism. Psychol. Rev. 114, 4, 864-886 (2007). https://doi.org/10.1037/0033-295X.114.4.864.

18. Følstad, A. et al.: What Makes Users Trust a Chatbot for Customer Service? An Exploratory Interview Study. In: Internet Science. INSCI 2018. Lecture Notes in Computer Science. (2018). https://doi.org/10.1007/978-3-030-01437-7_16.

19. Fournier, S.: Consumers and Their Brands: Developing Relationship Theory in Consumer Research. J. Consum. Res. 24, 4, 343-353 (1998). https://doi.org/10.1086/209515.

20. Go, E., Sundar, S.S.: Humanizing Chatbots: The effects of visual, identity and conversational cues on humanness perceptions. Comput. Human Behav. (2019). https://doi.org/10.1016/j.chb.2019.01.020. 
21. Griol, D. et al.: An automatic dialog simulation technique to develop and evaluate interactive conversational agents. Appl. Artif. Intell. 27, 9, 759-780 (2013). https://doi.org/10.1080/08839514.2013.835230.

22. Guzman, A.L.: Voices in and of the machine: Source orientation toward mobile virtual assistants. Comput. Human Behav. 90, 343-350 (2019). https://doi.org/10.1016/j.chb.2018.08.009.

23. Hassanein, K., Head, M.: Manipulating perceived social presence through the web interface and its impact on attitude towards online shopping. Int. J. Hum. Comput. Stud. 65, 8, 689-708 (2007). https://doi.org/10.1016/j.ijhcs.2006.11.018.

24. Hayes, A.F.: PROCESS: A versatile computational tool for observed variable mediation, moderation, and conditional process modeling. White Pap. 1-39 (2012). https://doi.org/978-1-60918-230-4.

25. Hayes, A.F., Preacher, K.J.: Statistical mediation analysis with a multicategorical independent variable. Br. J. Math. Stat. Psychol. 67, 3, 451-470 (2014). https://doi.org/10.1111/bmsp.12028.

26. Ischen, C. et al.: How important is agency? The persuasive consequences of interacting with a chatbot as a new entity. Pap. Present. Human-Machine Commun. ICA PreConference, Washington, D.C. (2019).

27. Kim, Y., Sundar, S.S.: Anthropomorphism of computers: Is it mindful or mindless? Comput. Human Behav. 28, 1, 241-250 (2012). https://doi.org/10.1016/j.chb.2011.09.006.

28. Ledbetter, A.M.: Measuring online communication attitude: Instrument development and validation. Commun. Monogr. 76, 4, 463-486 (2009). https://doi.org/10.1080/03637750903300262.

29. Marathe, S. et al.: Who are these power users anyway? Building a psychological profile. (2007).

30. Nass, C., Moon, Y.: Machines and Mildlessness: Social Responses to Computers. J. Soc. Issues. 56, 1, 86-103 (2000).

31. van Noort, G., Willemsen, L.M.: Online Damage Control: The Effects of Proactive Versus Reactive Webcare Interventions in Consumer-generated and Brand-generated Platforms. J. Interact. Mark. 26, 3, 131-140 (2012). https://doi.org/10.1016/j.intmar.2011.07.001.

32. Powers, A., Kiesler, S.: The advisor robot: Tracing people's mental model from a robot's physical attributes. Proc. 1st ACM SIGCHI/SIGART Conf. Human-robot Interact. 218-225 (2006). https://doi.org/10.1145/1121241.1121280.

33. Reeves, B., Nass, C.: The media equation: How people treat computers, television, and new media like real people and places. Cambrigde University Press (1996).

34. Sundar, S.S., Kim, J.: Machine Heuristic: When We Trust Computers More than Humans with Our Personal Information. Proc. 2019 CHI Conf. Hum. Factors Comput. Syst. - CHI '19. 1-9 (2019). https://doi.org/10.1145/3290605.3300768.

35. Sundar, S.S., Nass, C.: Source orientation in human-computer interaction: Programmer, networker, or independent social actor. Communic. Res. 27, 6, 683-703 (2000).

36. Verhagen, $\mathrm{T}$. et al.: Virtual customer service agents: Using social presence and personalization to shape online service encounters. J. Comput. Commun. 19, 3, 529545 (2014). https://doi.org/10.1111/jcc4.12066. 
37. Westin, A.F.: Privacy and freedom. Wash. Lee Law Rev. 25, 1, 166 (1967).

38. Whang, C.: Voice Shopping: The Effect of the Consumer-Voice Assistant Parasocial Relationship on the Consumer's Perception and Decision Making. (2018).

39. Wottrich, V.M. et al.: The role of customization, brand trust, and privacy concerns in advergaming. Int. J. Advert. 36, 1, 60-81 (2017). https://doi.org/10.1080/02650487.2016.1186951.

40. $\mathrm{Xu}, \mathrm{H}$. et al.: Examining the Formation of Individual's Privacy Concerns: Toward an Integrative View. Int. Conf. Inf. Syst. Proc. 1-16 (2008). https://doi.org/citeulikearticle-id:5770148.

41. Zajonc, R.B.: Mere exposure: A gateway to the subliminal. Curr. Dir. Psychol. Sci. 10, 6, 224-228 (2001). https://doi.org/10.1111/1467-8721.00154.

42. Zarouali, B. et al.: Predicting Consumer Responses to a Chatbot on Facebook. Cyberpsychology, Behav. Soc. Netw. 21, 8, cyber.2017.0518 (2018). https://doi.org/10.1089/cyber.2017.0518.

43. Zhou, L. et al.: Non-local or local brands? A multi-level investigation into confidence in brand origin identification and its strategic implications. J. Acad. Mark. Sci. 38, 2, 202-218 (2010). https://doi.org/10.1007/s11747-009-0153-1.

Acknowledgements. This study was funded by the Research Priority Area Communication and its Digital Communication Methods Lab (digicomlab.eu) at the University of Amsterdam. 